\title{
Study of Intermodulation in RF MEMS Variable Capacitors
}

\author{
David Girbau, Student Member, IEEE, Nerea Otegi, Lluís Pradell, Member, IEEE, and Antonio Lázaro
}

\begin{abstract}
This paper provides a rigorous study of the causes and physical origins of intermodulation distortion (IMD) in RF microelectromechanical systems (MEMS) capacitors, its analytical dependence on the MEMS device design parameters, and its effects in RF systems. It is shown that not only third-order products exist, but also fifth order and higher. The high-order terms are mainly originated by the nonlinear membrane displacement versus applied voltage and, in the case considered in this study, with an additional contribution from the nonlinear dependence of the reflection coefficient phase on the displacement. It is also shown that the displacement nonlinear behavior also contributes to the total mean position of the membrane. In order to study these effects in depth, an analytical frequency-dependent IMD model for RF MEMS based on a mobile membrane is proposed and particularized to the case of a MEMS varactor-a device for which IMD can be significant. The model is validated, up to the fifth order, theoretically (using harmonic balance) and empirically (the IMD of a MEMS varactor is measured). To this end, a two-tone IMD reflection measurement system for MEMS is proposed.
\end{abstract}

Index Terms-Intermodulation distortion (IMD), microelectromechanical systems (MEMS), MEMS varactor, self-actuation, two-tone IMD measurement.

\section{INTRODUCTION}

$\mathbf{M}$ ICROWAVE microelectromechanical systems (MEMS) have the chance of being important elements in the next future RF communication systems. MEMS switches and capacitors have already demonstrated to provide an important added value to circuits such as matching networks [1], phase shifters [2], [3], voltage-controlled oscillators [4], filters [5], antennas [6], [7], and impedance tuners [8]. The main advantages of replacing conventional switches and varactors with their MEMS counterparts are cost, size, and weight reduction, in addition to an excellent electrical performance-low loss and high quality factor [9], [10] — and the reconfigurability they provide to systems[11].

Some of the former circuits may work under a moderate-tohigh RF powercondition (e.g., reconfigurable matching networks in power amplifiers or switchable filters in transmitters) and, thus,

Manuscript received July 4, 2005; revised November 16, 2005. This work was supported by the Ministerio de Ciencia y Tecnología under Spanish Government Project ESP2004-07067-C03-03.

D. Girbau and L. Pradell are with the Department of Signal Theory and Communications, Universitat Politècnica de Catalunya, 08034 Barcelona, Spain (e-mail: DGirbauSala@tsc.upc.edu; pradell@tsc.upc.edu).

N. Otegi is with the Electricity and Electronics Department, University of the Basque Country, 48080 Bilbao, Spain (e-mail: weboturn@1g.ehu.es).

A. Lázaro is with the Electronics, Electrical, and Automatics Engineering Department, University Rovira i Virgili, 43007 Tarragona, Spain (e-mail: antonioramon.lazaro@urv.net).

Digital Object Identifier 10.1109/TMTT.2005.864116 the MEMS devices are also affected by the RF power. From the microwave circuit designer point-of-view, one undesired effect of RF power on MEMS is intermodulation distortion (IMD), which limits the maximum power a transmitter can deliver due to in-channel and cross-channel interference. Until not long ago, RF MEMS were assumed to be intermodulation-free devices. Although RF MEMS offer lower IMD than their semiconductor equivalents, this parameter cannot be ignored in MEMS devices under some excitation conditions. In the case of MEMS capacitive switches, it has been demonstrated that IMD is very small [12]. In addition, it is known that self-actuation is more critical in MEMS capacitors than in capacitive switches due to its larger area and the smaller gap required to achieve a nominal capacity (membrane in its up state), normally much higher than in capacitive switches. Consequently, since both self-actuation and IMD phenomena depend on the RF voltage at the device, it could reasonably be expected that the IMD in variable two parallel-plate capacitors would be more significant than in capacitive switches. This is because of their high nominal capacity, high RF voltage (due to standing waves at the capacitor in a shunt configuration), and a membrane positioning in highly nonlinear regions.

A number of models for IMD have been presented in the literature. In [12], an analytical model for third-order IMD in MEMS switches and an ADS-based nonlinear computeraided design (CAD) model, are proposed and compared to measurements of third-order IMD. The analytical model in [12] is based on two main approximations, i.e., small displacement of the membrane (linear zone) and high bridge impedance. However, these two approximations cannot be assumed in two parallel-plate capacitors because displacements up to $33 \%$ of the initial gap are expected and their higher nominal capacity corresponds to an impedance that cannot be considered as an open circuit. The model also assumes that the difference frequency between RF tones is much smaller than the device mechanical resonance frequency. A different approach [13], formulated in the frequency domain, is based on Volterra series and restricted to weak nonlinearities. It obtains simulated third-order IMD products in MEMS capacitors. A theoretical approximation to nonlinear effects in MEMS switches is presented in [14], while a two-tone mixed-domain simulation tool is applied to the study of distortion in MEMS capacitive shunt switches in [15]. Recently, a theoretical analysis of the distortion level in a resistive-contact MEMS switch has been presented [16]. In this case, the IMD in the up-state is not significant because of a small overlapping between plates; the IMD in the down state is modeled by means of a resistance variation due to the resistivity dependence on the temperature, which is a function of the RF tones. Another recent study [17] presents an extended 
harmonic-balance formulation to describe MEMS nonlinearities, providing a set of complementary equations to describe the nonlinear dynamics of MEMS and a number of techniques to solve convergence problems. In [18], a harmonic-balance analysis is applied to the nonlinear modeling of MEMS in reconfigurable antennas. In a previous study by the authors [19], a numerical model for a fast prediction of two-tone IMD in MEMS capacitors is proposed, but restricted to a small separation between tone frequencies (much less than the membrane resonance frequency). Measurements of IMD produced by a MEMS varactor for a two-tone test and digitally modulated signals are provided.

The aim of this paper is to propose an analytical model for computing the IMD originated in MEMS devices made up of two parallel plates and to provide a physical explanation of its origins. To this end, a general analysis of the mobile membrane displacement generated by two RF tones is developed. A solution for the one-dimensional (1-D) nonlinear differential equation ruling the membrane movement (mass-spring-damper) is proposed. The analysis is neither restricted to the linear zone, nor in frequency, as membrane displacement is frequency dependent. It must be stressed that, while an analytical model in the time domain is mandatory to relate IMD to its physical origins, its frequency dependence is also important to determine the influence of the external conditions (RF signal frequency) on IMD distortion. The proposed model is versatile and easy to use by the microwave designer because it provides the analytical dependence of the IMD products on design parameters in a closed form, as well as a quantitative description of the various nonlinear phenomena. It is theoretically demonstrated and empirically validated that, in MEMS capacitors, not only third-order intermodulation products are generated, but also fifth order (although higher order IMD products are also generated, their power is beyond our measurement limit). These products are generated by the nonlinear dependence of the membrane displacement on the applied voltage, and enhanced by the nonlinear nature of the reflection coefficient phase with the displacement. The contribution of the nonlinear terms to the membrane mean position is also analyzed. It is demonstrated that its effect is an enlargement of the mean displacement obtained from dc bias and self-actuation producing, in turn, an increase in all IMD products.

\section{Membrane Displacement Due to the Presence of Two RF TONES AND BIAS SignAL}

The dynamics of motion of a suspended membrane can be predicted using the 1-D nonlinear differential equation of a mass-spring-damper second-order system, which, assuming a two parallel-plate topology and electrostatic actuation, can be expressed as

$$
m \frac{d^{2} x}{d t^{2}}+b \frac{d x}{d t}+k x=\frac{1}{2} \frac{\varepsilon A V^{2}}{(d-x)^{2}}
$$

where (see Fig. 1) $x$ is the displacement, $d$ is the initial gap, $A$ is the electrodes' area (an effective equivalent area, which takes into account the fringing capacity: see Section VI-A), $V$ is the

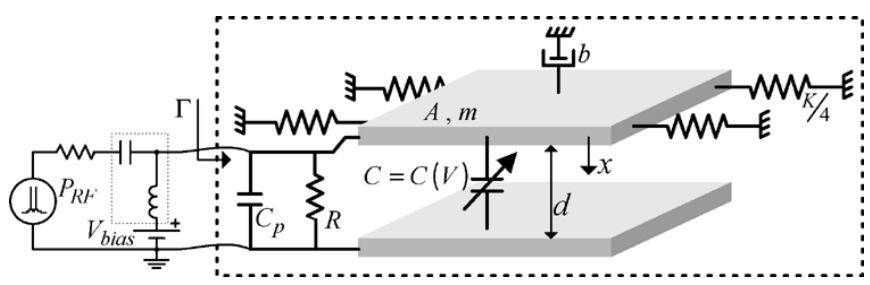

Fig. 1. Mechanical and electrical model of a two parallel-plate RF MEMS capacitor.

total applied voltage, and $\varepsilon$ is the air permittivity; $m, b$, and $k$ are the mass, damping coefficient, and total suspension stiffness constant of the mobile plate, respectively, as shown in Fig. 1.

Defining a signal at the capacitor composed of two RF tones and de bias

$$
V=V_{\text {bias }}+V_{1} \sin \omega_{1} t+V_{2} \sin \omega_{2} t
$$

the square of voltage $V$ in (1) reduces to

$$
V^{2} \approx V_{\text {bias }}^{2}+\frac{V_{1}^{2}}{2}+\frac{V_{2}^{2}}{2}+V_{1} V_{2} \cos \left(\omega_{2}-\omega_{1}\right) t
$$

where the high-frequency terms have been omitted since, due to the low-pass behavior of (1) $\left(\omega_{0}=\sqrt{k / m} \ll \omega_{1}, \omega_{2} ; \omega_{0}\right.$ being its mechanical resonance frequency), they have no influence on the membrane position. It must be stressed that $V_{1}$ and $V_{2}$ are the total voltages at the capacitor, which depend on the amplitudes of the incident waves $\left(V_{01}^{+}, V_{02}^{+}\right)$, the specific location of the device in the circuit and the device electrical parameters (reflection coefficient). This is an important difference in comparison to terminated MEMS switches, where the total RF voltage at the device is approximately the amplitude of the incident wave. Defining

$$
V_{\mathrm{DC}}=V_{\mathrm{bias}}^{2}+\frac{V_{1}^{2}}{2}+\frac{V_{2}^{2}}{2} \quad V_{t}=V_{1} V_{2}
$$

and the frequency difference between tones

$$
\omega=\omega_{2}-\omega_{1}
$$

$V^{2}$ in (1) is expressed as

$$
V^{2} \approx V_{\mathrm{DC}}+V_{t} \cos (\omega t)
$$

This means that the electrostatic force is proportional to a squared voltage composed of a dc component-caused by dc bias and self-actuation-and a time-varying component. Consequently, a constant shift plus a certain time-dependent displacement can be expected in the membrane response. In order to approximate the solution of (1) for voltage (2), a displacement composed of two parts, i.e., a constant dc part [due to the dc force component, which includes the rectified RF tones, as can be seen in (4)] and a time-varying one is proposed: $x=$ $x_{\mathrm{DC} 0}+x_{t}(t)$. Substituting this expression in (1), the following differential equation is obtained:

$$
\begin{aligned}
{\left[m \frac{d^{2} x_{t}}{d t^{2}}+b \frac{d x_{t}}{d t}+k x_{t}+k x_{\mathrm{DC} 0}\right] } & \left(1-\frac{x_{t}}{d-x_{\mathrm{DC} 0}}\right)^{2} \\
= & \frac{1}{2} \frac{\varepsilon A\left(V_{\mathrm{DC}}+V_{t} \cos \omega t\right)}{\left(d-x_{\mathrm{DC} 0}\right)^{2}} .
\end{aligned}
$$


Expanding (7) yields

$$
\begin{aligned}
m \ddot{x}_{t}+ & b \dot{x}_{t}+k_{2} x_{t}-\lambda x_{t}^{2}-2 \frac{x_{t}}{\left(d-x_{\mathrm{DC} 0}\right)}\left(m \ddot{x}_{t}+b \dot{x}_{t}\right) \\
& +\left(\frac{x_{t}}{\left(d-x_{\mathrm{DC} 0}\right)}\right)^{2}\left(m \ddot{x}_{t}+b \dot{x}_{t}\right)+k \frac{x_{t}^{3}}{\left(d-x_{\mathrm{DC} 0}\right)^{2}}+k x_{\mathrm{DC} 0} \\
= & F_{\mathrm{DC}}+F_{\omega} \cos (\omega t)
\end{aligned}
$$

where $\dot{x}_{t}$ and $\ddot{x}_{t}$ are first and second derivatives of $x_{t}(t)$, respectively; constants $k_{2}$ and $\lambda$ are defined as

$$
\begin{aligned}
k_{2} & =k\left(1-\frac{2 x_{\mathrm{DC} 0}}{d-x_{\mathrm{DC} 0}}\right) \\
\lambda & =k\left(\frac{2}{d-x_{\mathrm{DC} 0}}-\frac{x_{\mathrm{DC} 0}}{\left(d-x_{\mathrm{DC} 0}\right)^{2}}\right) .
\end{aligned}
$$

$F_{\omega}$ is the amplitude of the force ac component

$$
F_{\omega}=\frac{1}{2} \frac{\varepsilon A V_{t}}{\left(d-x_{\mathrm{DC} 0}\right)^{2}}
$$

and $F_{\mathrm{DC}}$ is the amplitude of the force dc component

$$
F_{\mathrm{DC}}=\frac{1}{2} \frac{\varepsilon A V_{\mathrm{DC}}}{\left(d-x_{\mathrm{DCO}}\right)^{2}} .
$$

In (8), the cubic term can be neglected because $x_{t}$ is very small. Furthermore, the cross-products between $x_{t}(t)$ and its derivatives can also be neglected in front of the linear and quadratic terms because they are multiplied by the parameters $m$ or $b$, which are much smaller than $k$. Applying the above approximations to (8), we obtain

$$
m \frac{d^{2} x_{t}}{d t^{2}}+b \frac{d x_{t}}{d t}+k_{2} x_{t}+k x_{\mathrm{DC} 0}=F_{\mathrm{DC}}+F_{\omega} \cos \omega t+\lambda x_{t}^{2}
$$

where the dc components of displacement and force $x_{\mathrm{DC} 0}$ and $F_{\mathrm{DC}}(12)$ satisfy the relation

$$
k x_{\mathrm{DC} 0}=F_{\mathrm{DC}}=\frac{1}{2} \frac{\varepsilon A V_{\mathrm{DC}}}{\left(d-x_{\mathrm{DC} 0}\right)^{2}} .
$$

Equation (13) can then be written as

$$
L\left(x_{t}\right)=m \frac{d^{2} x_{t}}{d t^{2}}+b \frac{d x_{t}}{d t}+k_{2} x_{t}=F_{\omega} \cos \omega t+\lambda x_{t}^{2} .
$$

A solution for the displacement $x_{t}(t)$ can be found using a Fourier series expansion

$$
x_{t}(t)=A_{0}+\sum_{n=1}^{N} A_{n \omega} \cos \left(n \omega t-\delta_{n \omega}\right)=\sum_{n=-N}^{N} c_{n} e^{j n \omega t} .
$$

The Fourier coefficients satisfy $c_{-n}=c_{n}^{*}$. Substituting (16) into (15), we obtain

$$
\begin{aligned}
& \sum_{n=-N}^{N}\left(-n^{2} \omega^{2} m+j \omega n b+k_{2}\right) c_{n} e^{j n \omega t} \\
& =\frac{1}{2} F_{\omega} e^{j \omega t}+\frac{1}{2} F_{\omega} e^{-j \omega t}+\lambda \sum_{n=-N}^{N} \sum_{l=-N}^{N} c_{n} c_{l} e^{j(n+l) \omega t}
\end{aligned}
$$

An analytical solution for the displacement $x_{t}$ [Fourier coefficients $c_{0}, c_{1}, c_{2}, \ldots, c_{N}$ in (16)] can be obtained by using the orthogonality property of the Fourier series in (17) for a convenient truncation order $N$. In this study, only the constant term $\left(c_{0}\right)$ and the first and second harmonics $c_{1}$ and $c_{2}$ are considered $(N=2)$. The reason is that higher order harmonics have much lower amplitude and their effect in the phase modulation can be neglected (see Section IV). Moreover, the dominant term in the response of the differential equation (15) is the forced term $c_{1}$ corresponding to the response in the linear region and, therefore, $\left|c_{1}\right| \gg c_{0},\left|c_{2}\right|$ can be assumed. With the above considerations, the following analytical approximations for the Fourier coefficients are obtained.

- DC term $(n=0)$

$$
\begin{aligned}
L\left(c_{0}\right) & =k_{2} c_{0}=\lambda\left|c_{0}\right|^{2}+2 \lambda\left(\left|c_{1}\right|^{2}+\left|c_{2}\right|^{2}\right) \approx 2 \lambda\left|c_{1}\right|^{2} \\
c_{0} & \approx \frac{2 \lambda}{k_{2}}\left|c_{1}\right|^{2} .
\end{aligned}
$$

- First harmonic $(n=1)$

$$
\begin{aligned}
L\left(c_{1} e^{j \omega t}\right) & =\left(-m \omega^{2}+b j \omega+k_{2}\right) c_{1} e^{j \omega t} \\
& =\frac{1}{2} F_{\omega} e^{j \omega t}+2 \lambda\left(c_{2} c_{-1}+c_{0} c_{1}\right) e^{j \omega t} \\
& \approx \frac{1}{2} F_{\omega} e^{j \omega t} .
\end{aligned}
$$

- Second harmonic $(n=2)$

$$
\begin{aligned}
L\left(c_{2} e^{j 2 \omega t}\right) & =\left(-4 m \omega^{2}+j 2 b \omega+k_{2}\right) c_{2} e^{j 2 \omega t} \\
& =\lambda\left(2 c_{0} c_{2}+c_{1}^{2}\right) e^{j 2 \omega t} \approx \lambda c_{1}^{2} e^{j 2 \omega t} .
\end{aligned}
$$

Using (16),

$$
\begin{aligned}
A_{\omega} & =2\left|c_{1}\right|=\frac{F_{\omega}}{\sqrt{\left(k_{2}-m \omega^{2}\right)^{2}+(b \omega)^{2}}} \\
\delta_{\omega} & =-\operatorname{angle}\left(c_{1}\right)=\operatorname{atan}\left(\frac{b \omega}{k_{2}-m \omega^{2}}\right) \\
A_{2 \omega} & =2\left|c_{2}\right|=\frac{\lambda\left|c_{1}\right|^{2}}{\sqrt{\left(k_{2}-4 m \omega^{2}\right)^{2}+(2 b \omega)^{2}}} \\
\delta_{2 \omega} & =-\operatorname{angle}\left(c_{2}\right)=2 \delta_{\omega}+\operatorname{atan}\left(\frac{2 b \omega}{k_{2}-4 m \omega^{2}}\right) .
\end{aligned}
$$


Note that the frequency response of the mass-spring-damper system at harmonic frequencies $\omega$ and $2 \omega$ [(22) and (24) respectively] has a low-pass behavior. Also note that a new contribution to the dc term $A_{0}=c_{0}$ emerges. The total dc component of displacement is then

$$
x_{\mathrm{DC}}=x_{\mathrm{DC} 0}+c_{0}=x_{\mathrm{DC} 0}+\frac{\lambda}{2 k_{2}} \frac{F_{\omega}^{2}}{\left(k_{2}-m \omega^{2}\right)^{2}+(b \omega)^{2}}
$$

and the global displacement can be split into a total dc component (or constant) $x_{\mathrm{DC}}(26)$ and a total time-varying component $x_{t-\text { tot }}$

$$
\begin{aligned}
x & =x_{\mathrm{DC}}+x_{t \_ \text {tot }}(t) \\
& =x_{\mathrm{DC}}+A_{\omega} \cos \left(\omega t-\delta_{\omega}\right)+A_{2 \omega} \cos \left(2 \omega t-\delta_{2 \omega}\right) .
\end{aligned}
$$

The dc-displacement $x_{\mathrm{DC} 0}$ caused by the applied dc bias and self-actuation can be obtained from (14) and, substituting it into (9)-(11) and (18)-(25), the time-dependent contribution $x_{t-\text { tot }}$ of (27) is solved. The displacement $x_{\mathrm{DC} 0}$, in spite of being the main contributor to $x_{\mathrm{DC}}$, is not the total dc displacement of the membrane, as the time-dependent part of the proposed solution $x_{t}(t)$ also presents a constant displacement resulting from the membrane nonlinear behavior (nonlinear terms in the equation).

It can also be deduced from (9), (22), and (24) that the mechanical bandwidth, in terms of IMD, is not constant, decreasing with an increasing applied dc voltage (DC bias + self-actuation).

It must be stressed that this analysis of the displacement is valid for any mobile membrane in a two parallel-plate configuration with electrostatic actuation either in a switch or in a capacitor and for any initial gap, area, or other parameters. Below, this displacement will be used in the particular case of a MEMS capacitor.

\section{Physical Interpretation of the Membrane DISPLACEMENT: ORIGIN OF INTERMODULATION}

A qualitative analysis of IMD generation under different excitation conditions, based on the study of the membrane displacement presented in Section II, is presented here. Several physical interpretations, with a direct relation to the origin of IMD in MEMS devices, can be deduced. A quantitative derivation of IMD products obtained from the analysis of the reflection coefficient phase is given in Sections IV and V. Fig. 2(a) shows the membrane displacement as a function of the squared voltage. This plot can be qualitatively compared to the load line of a transistor, the main difference being that this curve is not linear in MEMS. Therefore, the amplitude of displacement $x$ depends not only on the amplitude of the temporal component $V_{t}$ of the squared voltage [see (6)], but also on the dc component $V_{\mathrm{DC}}$ (bias voltage and self-actuation). It can be observed that, for an applied signal such as shown in (2), the displacement will be a small-amplitude sinusoid when the MEMS is in the linear region of its tuning range, generating only third-order IMD. This sinusoid suffers a growing degradation when the device behavior becomes more nonlinear, thus higher frequency components appearing in the membrane displacement, which, in turn, generate higher order intermodulation products. Moreover, the amplitudes of the displacement harmonics increase with the

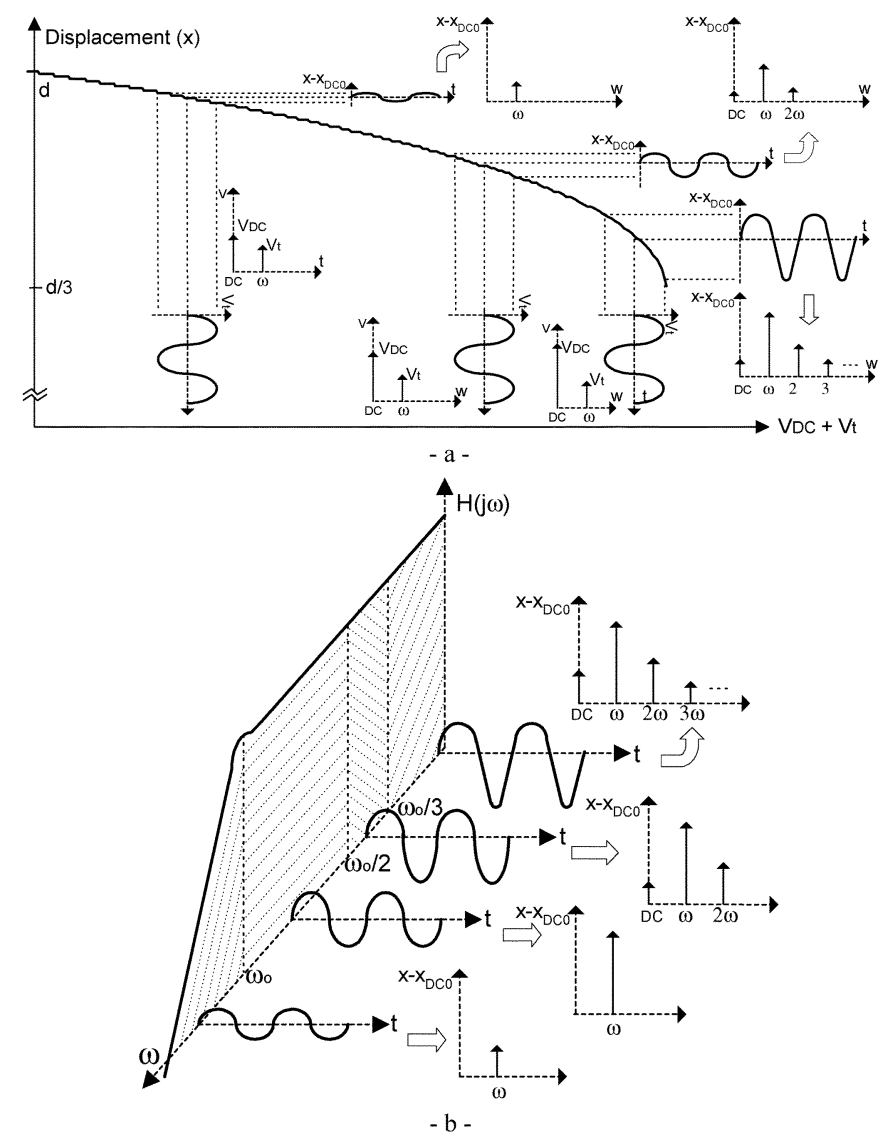

Fig. 2. (a) Generation of displacement harmonics due to the nonlinear behavior of the displacement as a function of the squared applied voltage. (b) Frequency dependence of the displacement harmonics due to the low-pass behavior of the membrane response.

TABLE I

FREQUENCIAL COMPONENTS IN DISPLACEMENT AND INTERMODULATION PRODUCTS

\begin{tabular}{lll}
\hline \hline$\omega$ & $\begin{array}{l}\text { Displacement } \\
\text { harmonics (spectrum) }\end{array}$ & $\begin{array}{l}\text { IMD products } \\
\text { generation }\end{array}$ \\
\hline$\omega<\omega_{0} / 3$ & $1^{\text {st }}, 2^{\text {nd }}, 3^{\text {rd }}$ & $3^{\text {rd }}, 5^{\text {th }}, 7^{\text {th }}$ \\
$\omega_{0} / 2>\omega>\omega_{0} / 3$ & $1^{\text {st }}, 2^{\text {nd }}$ & $3^{\text {rd }}, 5^{\text {th }}$ \\
& $3^{\text {rd }}$ attenuated & \\
$\omega_{o}>\omega>\omega_{0} / 2$ & $1^{\text {st }}$ & $3^{\text {rd }}$ \\
& $2^{\text {nd }}, 3^{\text {rd }}$, attenuated & $5^{\text {th }}$ attenuated \\
$\omega>\omega_{0}$ & $1^{\text {st }}$ attenuated & $3^{\text {rd }}$ attenuated \\
$\omega>>\omega_{0}$ & -- & -- \\
\hline
\end{tabular}

displacement nonlinearity, translating into an increase of power of all intermodulation products.

The analysis of Fig. 2(a) holds for the case $\omega \ll \omega_{0}$. As deduced from (27), the displacement is a function of frequency, due to the device low-pass behavior (1). Fig. 2(b) shows the frequency dependence for the worst case of Fig. 2(a), which corresponds to a highly nonlinear position. Depending on the frequency $\omega$, five different cases are defined. Table I summarizes the cases with their corresponding displacement harmonics and IMD generation. As it is demonstrated in Section IV, the intermodulation products are indeed generated by a modulation in the reflection-coefficient phase, which, in turn, is produced by the displacement harmonics. 

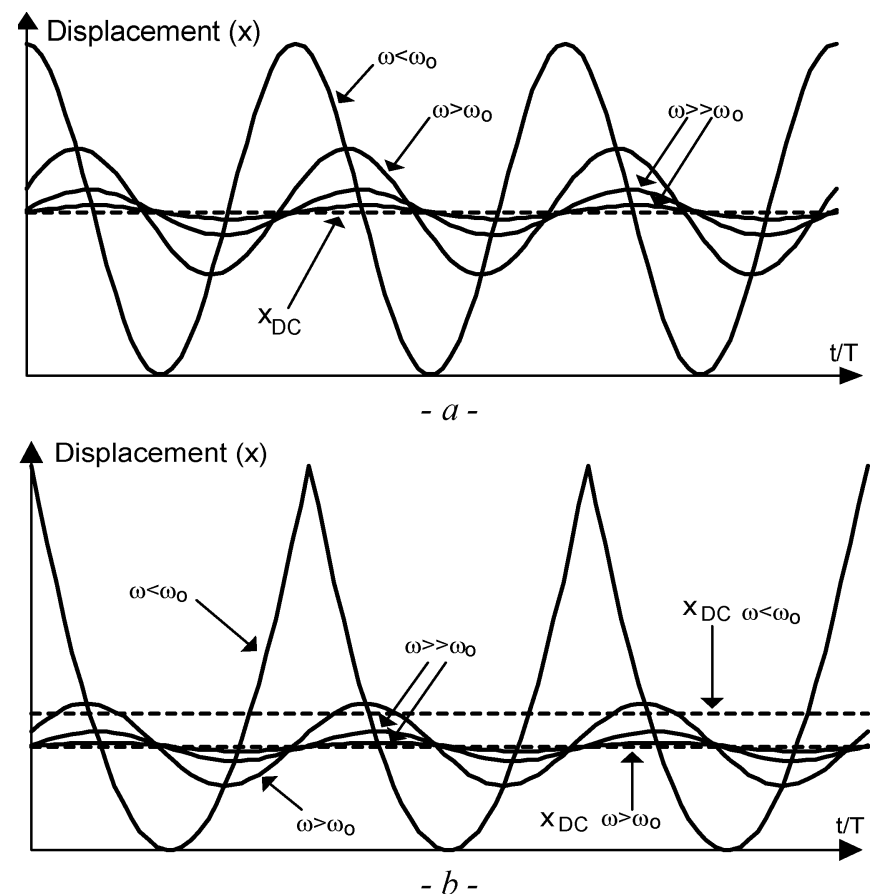

Fig. 3. Dependence of the membrane displacement on RF power at the capacitor. (a) Displacement for low RF power. (b) Displacement for high RF power.

It must be stressed that, in addition to the generation of higher order harmonics, an extra de displacement, which is significant for $\omega<\omega_{o} / 2$, arises and grows with an increasing displacement nonlinearity [see (26)]. As a conclusion of Fig. 2(b), the use of two tones with a frequency difference $\omega$ much larger than $\omega_{0}$ is recommendable. However, this is not always feasible as, for instance, in digitally modulated wide-band signals [19].

The dependence of displacement on the total applied voltage and on the frequency $\omega$ is observed in Fig. 3. Fig. 3(a) shows the displacement time waveforms of a membrane without dc bias, excited with a low RF power, for several RF tone separations $\omega$. It can be observed that the displacement has a sinusoidal behavior in all cases, showing decreasing amplitude for increasing $\omega$. It must be stressed that the displacement dc component $x_{\mathrm{DC}}$ remains nearly constant in this case. Also note that, for $\omega<\omega_{0}$, the amplitude of the time-varying displacement agrees with the value of the displacement due to self-actuation.

On the other hand, Fig. 3(b) shows a case with high RF power and no dc bias. In this case, the squared voltage has large amplitude. The displacement of the membrane is plotted for several $\omega$ situations. It can be seen that the sinusoid suffers a distortion (higher harmonics appear) for the case of $\omega<\omega_{o}$, which is translated into fifth-order and higher order intermodulation products. Also note that, in this case, there is a significant constant displacement component, which adds to the initial value $x_{\mathrm{DC} 0}$ due to de bias and self-actuation.

Fig. 4 shows a similar situation as in Fig. 3(a), but adding a bias voltage for two cases: low bias voltage and high bias voltage. A low RF power is considered in both cases. In the case of a low-bias voltage, it can be seen that the membrane behavior is a sinusoid for any applied frequency. On the other hand, for a high-bias voltage (placing the membrane in a highly nonlinear position), the membrane describes a nonsinusoidal movement

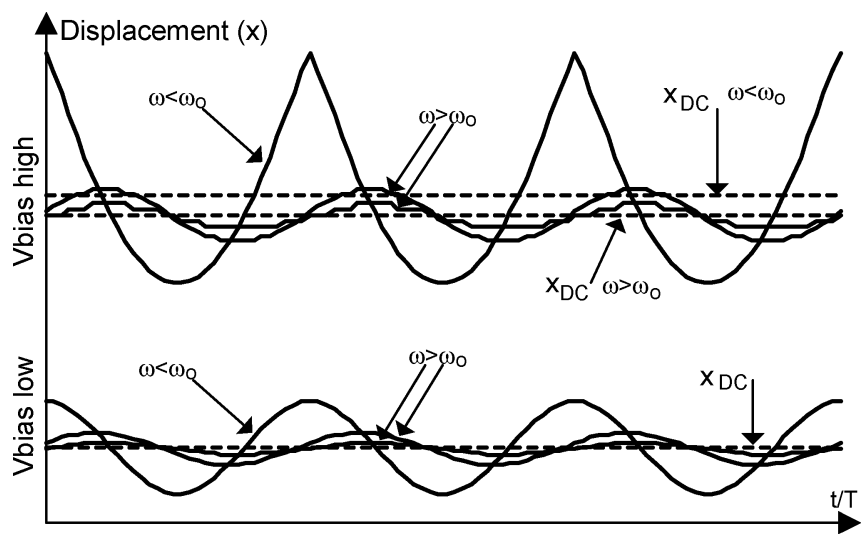

Fig. 4. Dependence of the membrane displacement on applied bias voltage for a low RF power.

and, therefore, fifth-order and higher intermodulation products are generated. In addition, there is an increase in the dc component because the nonlinear terms in the displacement equation are significant for high bias voltages.

\section{REFlection CoEfFicient Phase as a FunCtion OF THE MEMBRANE DiSPlACEMENT}

Since the device capacity depends on the instantaneous gap, the reflection coefficient is a function of the displacement. IMD is originated in the periodic phase variations caused by the instantaneous displacement studied in Sections II and III. The electrical model of the capacitor is the one proposed in Fig. 1, where a loss resistance $R$ and a parasitic capacity $C_{p}$ have been added for generalization purposes. The expressions presented here can be greatly simplified in low-loss devices or small parasitic capacity devices.

In the model of Fig. 1, the total capacity is

$$
C_{t o t}=C_{p}+C=C_{p}+\frac{\varepsilon A}{(d-x)}
$$

and the reflection coefficient for a pulsation $\omega_{i}(i=1,2)$ is

$$
\Gamma_{i}=\frac{R-Z_{0}-j Z_{0} \omega_{i}\left(C_{p}+\frac{\varepsilon A}{(d-x)}\right) R}{R+Z_{0}+j Z_{0} \omega_{i}\left(C_{p}+\frac{\varepsilon A}{(d-x)}\right) R} .
$$

Since the RF tone separation $\omega$ required for generation of displacement harmonics and IMD [see Fig. 2(b)] is comparable to the mechanical bandwidth of the device (this being in the order of kilohertz), it is assumed that the reflection coefficient is identical at both frequencies $\omega_{1}$ and $\omega_{2}$, namely, $\Gamma=\Gamma_{1} \approx \Gamma_{2}$.

Substituting $x$ in (29) with (27) and defining $d_{\mathrm{DC}}=d-x_{\mathrm{DC}}$, the reflection coefficient phase is

$$
\begin{aligned}
& \Phi=\angle \Gamma \\
& =a \tan \left(\frac{-2 Z_{0} \omega_{i}\left(C_{p}+\frac{\varepsilon A}{\left(d_{\mathrm{DC}}-x_{t_{-} \text {tot }}\right)}\right) R^{2}}{R^{2}-Z_{0}^{2}-\left(Z_{0} \omega_{i}\left(C_{p}+\frac{\varepsilon A}{\left(d_{\mathrm{DC}}-x_{t_{-} \text {tot }}\right)}\right) R\right)^{2}}\right) .
\end{aligned}
$$


Expanding (30) in a Taylor series (up to the quadratic term), the phase can be approximated as

$$
\Phi \approx \Phi(0)+\alpha x_{t_{-} \text {tot }}+\beta x_{t_{-} \text {tot }}^{2} \approx \Phi_{\mathrm{DC}}+\Phi_{t}
$$

where

$$
\begin{aligned}
\Phi_{\mathrm{DC}}= & \Phi(0)+\beta \frac{A_{\omega}^{2}}{2}+\beta \frac{A_{2 \omega}^{2}}{2} \\
\Phi_{t}= & \alpha A_{\omega} \cos \left(\omega t-\delta_{\omega}\right)+\beta A_{\omega} A_{2 \omega} \cos \left(\omega t+\delta_{\omega}-\delta_{2 \omega}\right) \\
& +\alpha A_{2 \omega} \cos \left(2 \omega t-\delta_{2 \omega}\right)+\beta \frac{A_{\omega}^{2}}{2} \cos \left(2 \omega t-2 \delta_{\omega}\right) \\
& +\beta A_{\omega} A_{2 \omega} \cos \left(3 \omega t-\delta_{\omega}-\delta_{2 \omega}\right) \\
& +\beta \frac{A_{2 \omega}^{2}}{2} \cos \left(4 \omega t-2 \delta_{2 \omega}\right)
\end{aligned}
$$

being

$$
\Phi(0)=\operatorname{atan}\left(\frac{-2 Z_{0} \omega_{i} R^{2}\left(C_{p}+\frac{\varepsilon A}{d_{\mathrm{DC}}}\right)}{\left(R^{2}-Z_{0}^{2}+\left(C_{p}+\frac{\varepsilon A}{d_{\mathrm{DC}}}\right)^{2} R^{2} \omega_{i}^{2} Z_{0}^{2}\right)}\right)
$$

and (35) and (36), shown at the bottom of this page.

In (32), it can be appreciated that two constant phase contributions, originated in the quadratic term of (31), add to $\Phi(0)$ - the phase at position $d_{\mathrm{DC}}$. It can also be observed in (31) and (33) that the reflection-coefficient phase is affected by a periodic modulation, this being the cause of intermodulation product generation. Note that the nonlinear dependence of phase on the displacement has been modeled up to the quadratic term. This approximation has proven to be sufficient to model intermodulation up to the fifth-order term.

Fig. 5 shows a schematic of all the contributions to IMD. First, the contribution of each displacement harmonic (whose generation has been studied in Section II) to the reflection-coefficient phase variation can be observed. In a second step, each phase harmonic is translated into its respective intermodulation tone, whose power will be determined in Section V.

\section{ANALYSIS OF INTERMOdUlation Product POWER}

In Section VI, the analytical model proposed in Sections II-V is validated by comparison to harmonic-balance simulations and

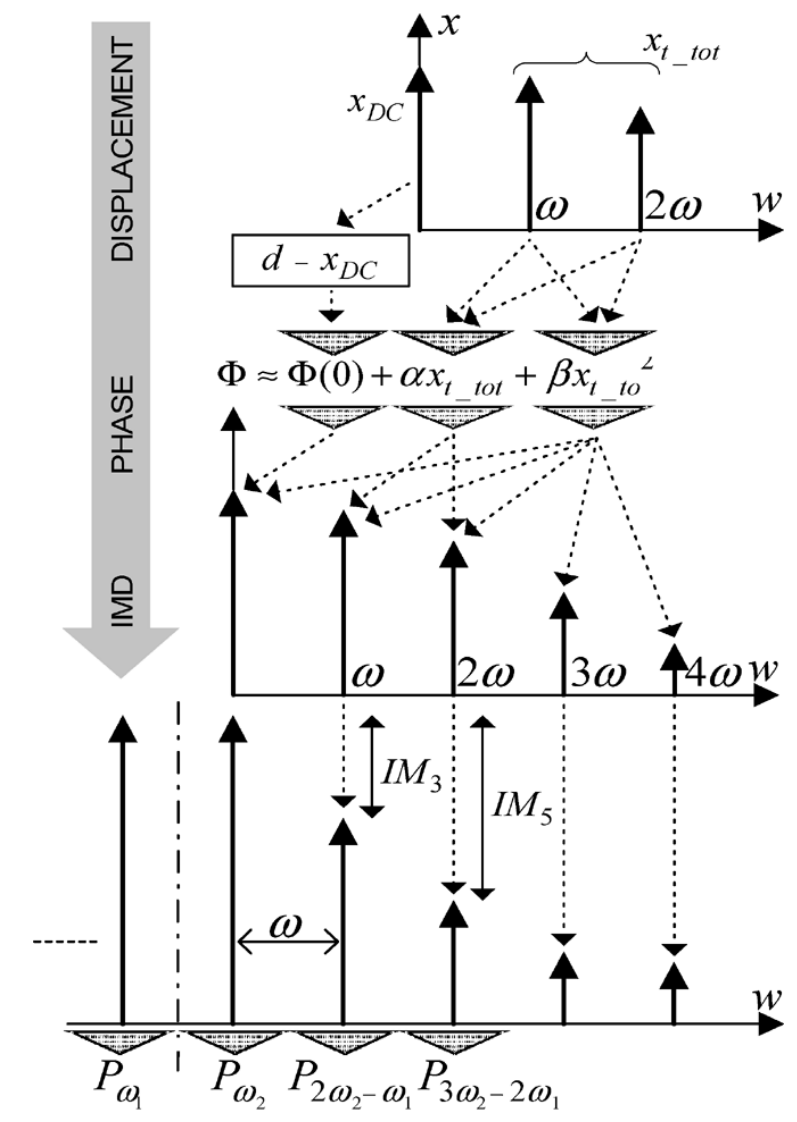

Fig. 5. Analysis of the contributions to intermodulation product generation.

to experimental results. To this end, an experimental setup for characterizing the IMD produced by MEMS capacitors is proposed. It is based on measuring the reflected waves from the MEMS device. Specifically, IMD is defined as the power ratio between the intermodulation products and the fundamental reflected waves (see Fig. 5). Here, the intermodulation product power, as well as third- and fifth-order IMD IM 3 and $\mathrm{IM}_{5}$ are obtained analytically.

The reflected signal at the MEMS capacitor can be expressed as

$$
V_{r}=|\Gamma| V_{01}^{+} \sin \left(\omega_{1} t+\Phi\right)+|\Gamma| V_{02}^{+} \sin \left(\omega_{2} t+\Phi\right)
$$

$$
\begin{aligned}
\alpha= & \frac{-2 \varepsilon A R^{2} \omega_{i} Z_{0}\left(R^{2}-Z_{0}^{2}+\left(C_{p}+\frac{\varepsilon A}{d_{\mathrm{DC}}}\right)^{2} R^{2} \omega_{i}^{2} Z_{0}^{2}\right)}{d_{\mathrm{DC}}^{2}\left(\left(R-Z_{0}\right)^{2}+\left(C_{p}+\frac{\varepsilon A}{d_{\mathrm{DC}}}\right)^{2} R^{2} \omega_{i}^{2} Z_{0}^{2}\right)\left(\left(R+Z_{0}\right)^{2}+\left(C_{p}+\frac{\varepsilon A}{d_{\mathrm{DC}}}\right)^{2} R^{2} \omega_{i}^{2} Z_{0}^{2}\right)} \\
\beta= & \frac{-2 \varepsilon A R^{2} \omega_{i} Z_{0}}{d_{\mathrm{DC}}^{3}\left(\left(R-Z_{0}\right)^{2}+\left(C_{p}+\frac{\varepsilon A}{d_{\mathrm{DC}}}\right)^{2} R^{2} \omega_{i}^{2} Z_{0}^{2}\right)^{2}\left(\left(R+Z_{0}\right)^{2}+\left(C_{p}+\frac{\varepsilon A}{d_{\mathrm{DC}}}\right)^{2} R^{2} \omega_{i}^{2} Z_{0}^{2}\right)^{2}} \\
& *\left\{\left(\left(R-Z_{0}\right)^{3}\left(R+Z_{0}\right)^{3}+\left(C_{p}+\frac{\varepsilon A}{d_{\mathrm{DC}}}\right)^{2} R^{2} \omega_{i}^{2} Z_{0}^{2}\left(4 C_{p} R^{4}+2 \frac{\varepsilon A}{d_{\mathrm{DC}}}\left(R^{2}-Z_{0}^{2}\right)^{2}-C_{p}\left(R^{2}+Z_{0}^{2}\right)^{2}\right)\right)\right. \\
& \left.\quad+\left(\left(C_{p}+\frac{\varepsilon A}{d_{\mathrm{DC}}}\right)^{4} R^{4} \omega_{i}^{4} Z_{0}^{4}\left(\left(3 C_{p}+\frac{\varepsilon A}{d_{\mathrm{DC}}}\right) R^{2}+\left(C_{p}+\frac{3 \varepsilon A}{d_{\mathrm{DC}}}+C_{p}\left(C_{p}+\frac{\varepsilon A}{d_{\mathrm{DC}}}\right)^{2} R^{2} \omega_{i}^{2}\right) Z_{0}^{2}\right)\right)\right\}
\end{aligned}
$$


where $V_{01}^{+}$and $V_{02}^{+}$are the amplitudes of the incident waves and $|\Gamma|$ is the magnitude of the reflection coefficient (29), which, in this study, is considered constant for all positions of the membrane. Indeed, as shown below, this parameter has no contribution to the calculation of $\mathrm{IM}_{3}$ and $\mathrm{IM}_{5}$. Using (31), the phase can be split into a constant and a time-dependent term $\Phi=\Phi_{\mathrm{DC}}+\Phi_{t}$. Considering $\Phi_{t}$ small enough, (37) can be approximated by a Taylor-series expansion as a function of $\Phi_{t}$ as follows:

$$
\begin{aligned}
V_{r} \approx|\Gamma| & {\left[V_{01}^{+} \sin \left(\omega_{1} t+\Phi_{\mathrm{DC}}\right)+V_{02}^{+} \sin \left(\omega_{2} t+\Phi_{\mathrm{DC}}\right)\right] } \\
& +|\Gamma| \Phi_{t}\left[V_{01}^{+} \sin \left(\omega_{1} t+\Phi_{\mathrm{DC}}\right)+V_{02}^{+} \sin \left(\omega_{2} t+\Phi_{\mathrm{DC}}\right)\right] .
\end{aligned}
$$

In (38), only the constant and linear terms of the Taylor series have been considered. Substituting $\Phi_{t}$ with (33) and neglecting those tones of order higher than fifth, the reflected signal, including the intermodulation terms corresponding to the upper sideband $V_{r_{-} \text {up }}$, can be expressed as follows in (39) (the lower sideband is identical provided that the amplitudes of the incident waves are the same):

$$
\begin{aligned}
V_{r_{-} \text {up }} \approx & |\Gamma| Q_{\omega_{2}}^{I} \sin \left(\omega_{2} t+\Phi_{\mathrm{DC}}\right)+|\Gamma| Q_{\omega_{2}}^{I I} \cos \left(\omega_{2} t+\Phi_{\mathrm{DC}}\right) \\
& +|\Gamma| Q_{2 \omega_{2}-\omega_{1}}^{I} \sin \left(\left(2 \omega_{2}-\omega_{1}\right) t+\Phi_{\mathrm{DC}}\right) \\
& +|\Gamma| Q_{2 \omega_{2}-\omega_{1}}^{I I} \cos \left(\left(2 \omega_{2}-\omega_{1}\right) t+\Phi_{\mathrm{DC}}\right) \\
& +|\Gamma| Q_{3 \omega_{2}-2 \omega_{1}}^{I} \sin \left(\left(3 \omega_{2}-2 \omega_{1}\right) t+\Phi_{\mathrm{DC}}\right) \\
& +|\Gamma| Q_{3 \omega_{2}-2 \omega_{1}}^{I I} \cos \left(\left(3 \omega_{2}-2 \omega_{1}\right) t+\Phi_{\mathrm{DC}}\right)
\end{aligned}
$$

where

$$
\begin{aligned}
Q_{\omega_{2}}^{I}=( & V_{02}^{+}+\frac{1}{2} V_{01}^{+} \alpha A_{\omega} \sin \left(\delta_{\omega}\right) \\
& \left.-\frac{1}{2} V_{01}^{+} \beta A_{\omega} A_{2 \omega} \sin \left(\delta_{\omega}-\delta_{2 \omega}\right)\right) \\
Q_{\omega_{2}}^{I I}=( & \frac{1}{2} V_{01}^{+} \alpha A_{\omega} \cos \left(\delta_{\omega}\right) \\
& \left.+\frac{1}{2} V_{01}^{+} \beta A_{\omega} A_{2 \omega} \cos \left(\delta_{\omega}-\delta_{2 \omega}\right)\right) \\
Q_{2 \omega_{2}-\omega_{1}}^{I}=( & \frac{1}{2} V_{02}^{+} \alpha A_{\omega} \sin \left(\delta_{\omega}\right) \\
& -\frac{1}{2} V_{02}^{+} \beta A_{\omega} A_{2 \omega} \sin \left(\delta_{\omega}-\delta_{2 \omega}\right) \\
& \left.+\frac{1}{4} V_{01}^{+} \beta A_{\omega}^{2} \sin \left(2 \delta_{\omega}\right)+\frac{1}{2} V_{01}^{+} \alpha A_{2 \omega} \sin \left(\delta_{2 \omega}\right)\right) \\
Q_{2 \omega_{2}-\omega_{1}=}^{I I} & \frac{1}{2} V_{02}^{+} \alpha A_{\omega} \cos \left(\delta_{\omega}\right) \\
& +\frac{1}{2} V_{02}^{+} \beta A_{\omega} A_{2 \omega} \cos \left(\delta_{\omega}-\delta_{2 \omega}\right) \\
& \left.+\frac{1}{4} V_{01}^{+} \beta A_{\omega}^{2} \cos \left(2 \delta_{\omega}\right)+\frac{1}{2} V_{01}^{+} \alpha A_{2 \omega} \cos \left(\delta_{2 \omega}\right)\right) \\
Q_{3 \omega_{2}-2 \omega_{1}}= & \frac{1}{2} V_{02}^{+} \alpha A_{2 \omega} \sin \left(\delta_{2 \omega}\right)+\frac{1}{4} V_{02}^{+} \beta A_{\omega}^{2} \sin \left(2 \delta_{\omega}\right) \\
& \left.+\frac{1}{2} V_{01}^{+} \beta A_{\omega} A_{2 \omega} \sin \left(\delta_{\omega}+\delta_{2 \omega}\right)\right) \\
Q_{3 \omega_{2}-2 \omega_{1}=}^{I I}= & \frac{1}{2} V_{02}^{+} \alpha A_{2 \omega} \cos \left(\delta_{2 \omega}\right)+\frac{1}{4} V_{02}^{+} \beta A_{\omega}^{2} \cos \left(2 \delta_{\omega}\right) \\
& \left.+\frac{1}{2} \beta A_{\omega} A_{2 \omega} \cos \left(\delta_{\omega}+\delta_{2 \omega}\right)\right) .
\end{aligned}
$$

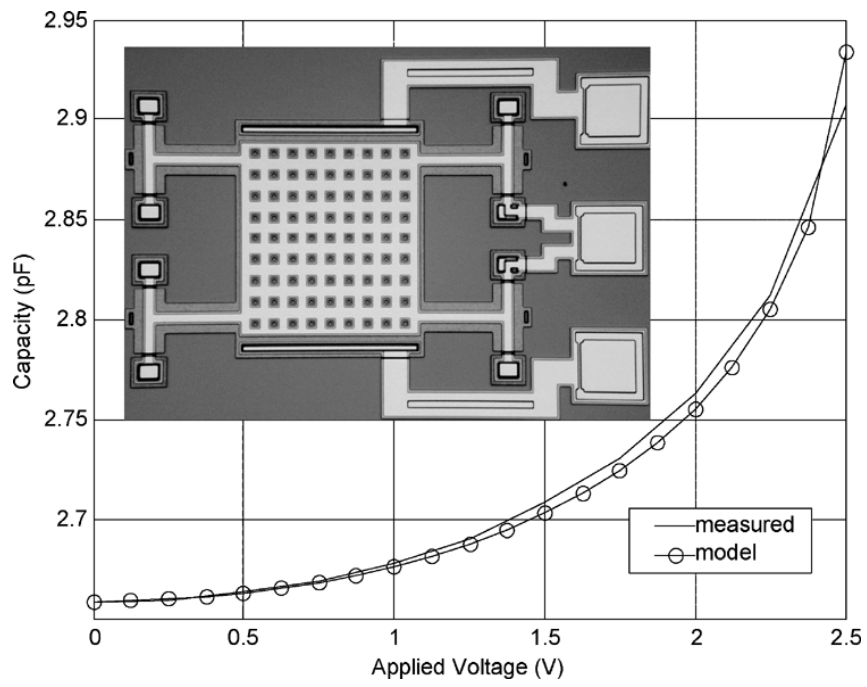

Fig. 6. Manufactured capacitor [20] and plots of the capacity versus voltage, measured and simulated.

Since $\alpha, \beta \ll 1$, the reflected voltage at the main frequency $\omega_{2}$ can be simplified to

$$
V_{r_{-} \text {up } \_\omega_{2}} \approx V_{02}^{+}|\Gamma| \sin \left(\omega_{2} t+\Phi_{\mathrm{DC}}\right) .
$$

The reflected power at the main frequency $\omega_{2}$ and the intermodulation product power can be derived from (42)-(44) as follows:

$$
\begin{aligned}
P_{r_{-} \omega_{2}} & =|\Gamma|^{2} \frac{\left(V_{02}^{+}\right)^{2}}{2 Z_{0}} \\
P_{r_{-} 2 \omega_{2}-\omega_{1}} & =|\Gamma|^{2} \frac{\left[\left(Q_{2 \omega_{2}-\omega_{1}}^{I}\right)^{2}+\left(Q_{2 \omega_{2}-\omega_{1}}^{I I}\right)^{2}\right]}{2 Z_{0}} \\
P_{r_{-} 3 \omega_{2}-2 \omega_{1}} & =|\Gamma|^{2} \frac{\left[\left(Q_{3 \omega_{2}-2 \omega_{1}}^{I}\right)^{2}+\left(Q_{3 \omega_{2}-2 \omega_{1}}^{I I}\right)^{2}\right]}{2 Z_{0}} .
\end{aligned}
$$

Finally, third- and fifth-order IMD can be calculated from (45) and (46), respectively, as follows:

$$
\begin{aligned}
& \mathrm{IM}_{3}(\mathrm{dBc})=P_{r_{-} 2 \omega_{2}-\omega_{1}}(\mathrm{~dB})-P_{r_{-} \omega_{2}}(\mathrm{~dB}) \\
& \mathrm{IM}_{5}(\mathrm{dBc})=P_{r_{-} 3 \omega_{2}-2 \omega_{1}}(\mathrm{~dB})-P_{r_{-} \omega_{2}}(\mathrm{~dB}) .
\end{aligned}
$$

It is observed that $\mathrm{IM}_{3}$ and $\mathrm{IM}_{5}$ do not depend on $|\Gamma|$. Note also that these IMD products are a function of the RF frequency due to the reflection coefficient phase frequency-dependence [see (30)].

\section{ANALYsis Validation: SimUlations AND MEASUREMENTS}

\section{A. Measured Device}

A two parallel-plate capacitor, previously designed and manufactured [20] (see a top view photograph in Fig. 6), has been used to validate the proposed analytical model (Sections II-V). Fig. 6 also shows a plot of the capacity versus voltage, simulated and measured. Curve fitting is crucial, as all the IMD calculations are based on the 1-D model (28). This model, along with (1), assumes the mobile plate to move as a rigid block with a uniformly distributed stiffness constant $(k)$, which is approximately the case for the low- $k$ device of Fig. 6 . For high- $k$ devices, the mobile plate suffers some bending, and the 1-D model 
TABLE II

PARAMETERS OF THE SiMULATED AND MEASURED CAPACITOR

\begin{tabular}{ccc}
\hline \hline Symbol & Parameter & Value \\
\hline$C$ & nominal capacity & $0.705 \mathrm{pF}$ \\
$C_{p}$ & parasitic capacity & $1.953 \mathrm{pF}$ \\
$A$ & effective area & $5.98 .10^{-8} \mathrm{~m}^{2}$ \\
$k$ & equivalent stiffness constant & $27 \mathrm{~N} / \mathrm{m}$ \\
$d$ & initial gap & $0.75 \mu \mathrm{m}$ \\
$R$ & loss resistance & $0.468 \mathrm{~K} \Omega$ \\
$Z_{0}$ & reference impedance & $50 \Omega$ \\
$m$ & mass & $0.199 \mathrm{ng}$ \\
$b$ & damping coefficient & $2.93 .10^{-4}$ \\
\hline
\end{tabular}

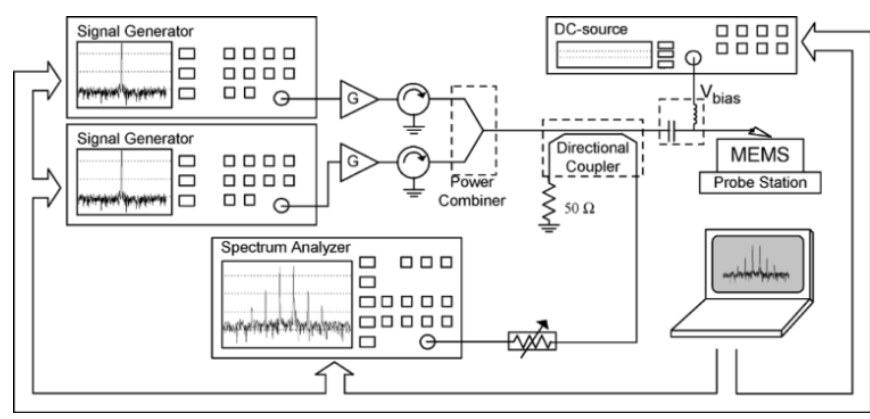

Fig. 7. Two-tone IMD measurement system for MEMS capacitors.

is no longer valid. In this case, the two plots differ, and the fitting of movement and capacity is performed through a reduction of the real model order by finding effective parameters for a correct prediction of IMD. Table II summarizes the main parameters of the simulated and measured capacitor manufactured using the surface micromachining PolyMUMPS process [21]. The effective area A takes into account the effect of the fringing capacity.

\section{B. Measurement Setup}

Fig. 7 shows the measurement system implemented to characterize two-tone IMD in MEMS capacitors. The system is designed to measure in reflection, a required feature for characterizing capacitors (one-port devices), in contrast to the one proposed in [12]. Two tones are injected into the capacitor using a power combiner. In order to provide enough isolation between both signal generators, an amplifier plus a circulator have been included in each path. A high isolation is essential to avoid injection locking between generators, as the difference frequency is very small, well inside the bandwidth of the loop filters in the synthesizers. A sample of the reflected signal in the MEMS device - which includes the generated IMD tones-is obtained through a directional coupler and displayed in a spectrum analyzer. In this way, IMD is measured in the reflected wave. All measurements in Section VI-C are performed according to this measurement concept.

\section{Simulations and Measurements}

Here, the proposed model is validated through measurements. Fig. 8 shows four spectra of the reflected signal, corresponding to four RF tone separations $\omega$. Fifth-order intermodulation is clearly observed for the cases of $\omega=100 \mathrm{~Hz}$ and $6 \mathrm{kHz}$. The IMD level is frequency dependent, as can be observed by a de-
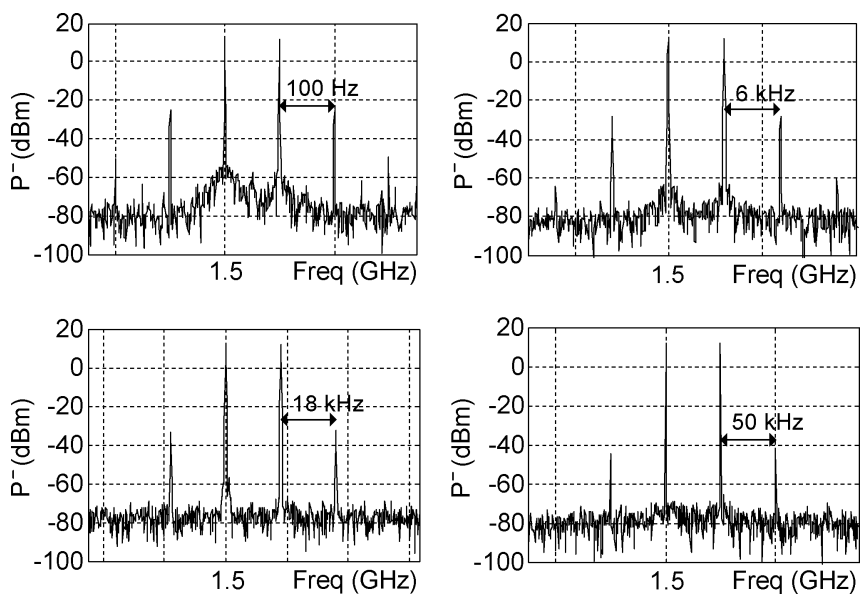

Fig. 8. Reflected signal spectrum for four frequency separations between RF tones $\omega(100 \mathrm{~Hz}$ and 6,18 , and $50 \mathrm{kHz})$. RF power is $P_{\mathrm{RF}}=12 \mathrm{dBm}$. A decrease in third- and fifth-order intermodulation products power with increasing $\omega$ is observed.

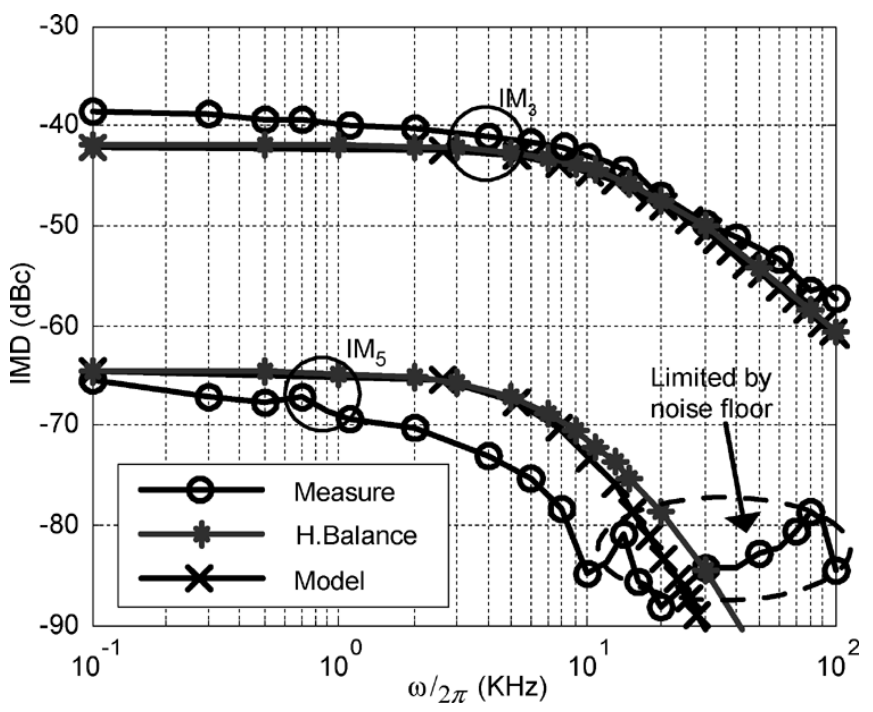

Fig. 9. Validation of the proposed analysis. Comparison with measurements and harmonic-balance simulations for the case of $P_{\mathrm{RF}}=11 \mathrm{dBm}$ as a function of $\omega$.

crease of the intermodulation product power with increasing RF tone frequency separation.

Fig. 9 shows a comparison of the results obtained with the proposed model to harmonic-balance simulations (based on the ADS nonlinear model of [12]), as well as to measured results. A very good agreement between models for predicting third- and fifth-order IMD is observed. Compared to measured results, it is observed that, while both models agree with measured thirdorder IMD, they predict, for the case of fifth order, a distortion worse than the measured one. This can be attributed to the fact that the measured capacitor does not behave exactly as predicted by the 1-D model, which assumes the membrane to move as a rigid block with a uniformly distributed restoring mechanical force. This means that the displacement sinusoid is not as much distorted as predicted.

The measurement of IMD has been performed in open air, as can be deduced from Fig. 9, where an overdamped behavior and, thus, no resonance frequency, is observed. 


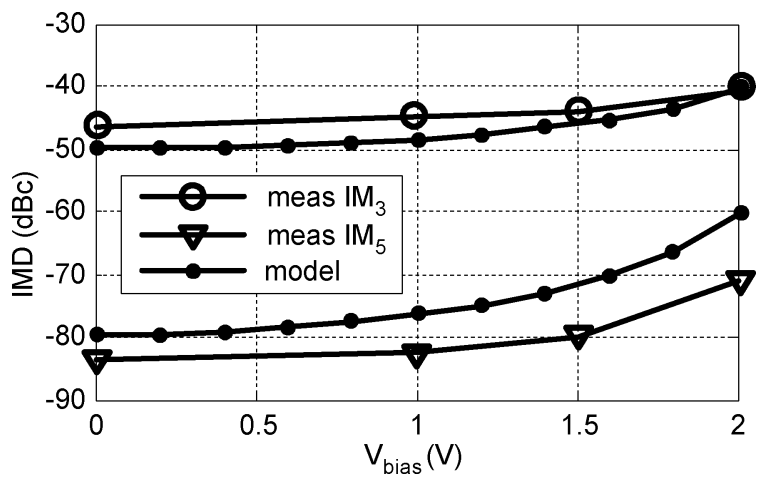

Fig. 10. Comparison of third- and fifth-order IMD between the proposed analytical model and measurements as a function of bias voltage $V_{\text {bias }}$. $P_{\mathrm{RF}}=8 \mathrm{dBm} . \omega / 2 \pi=100 \mathrm{~Hz}$.
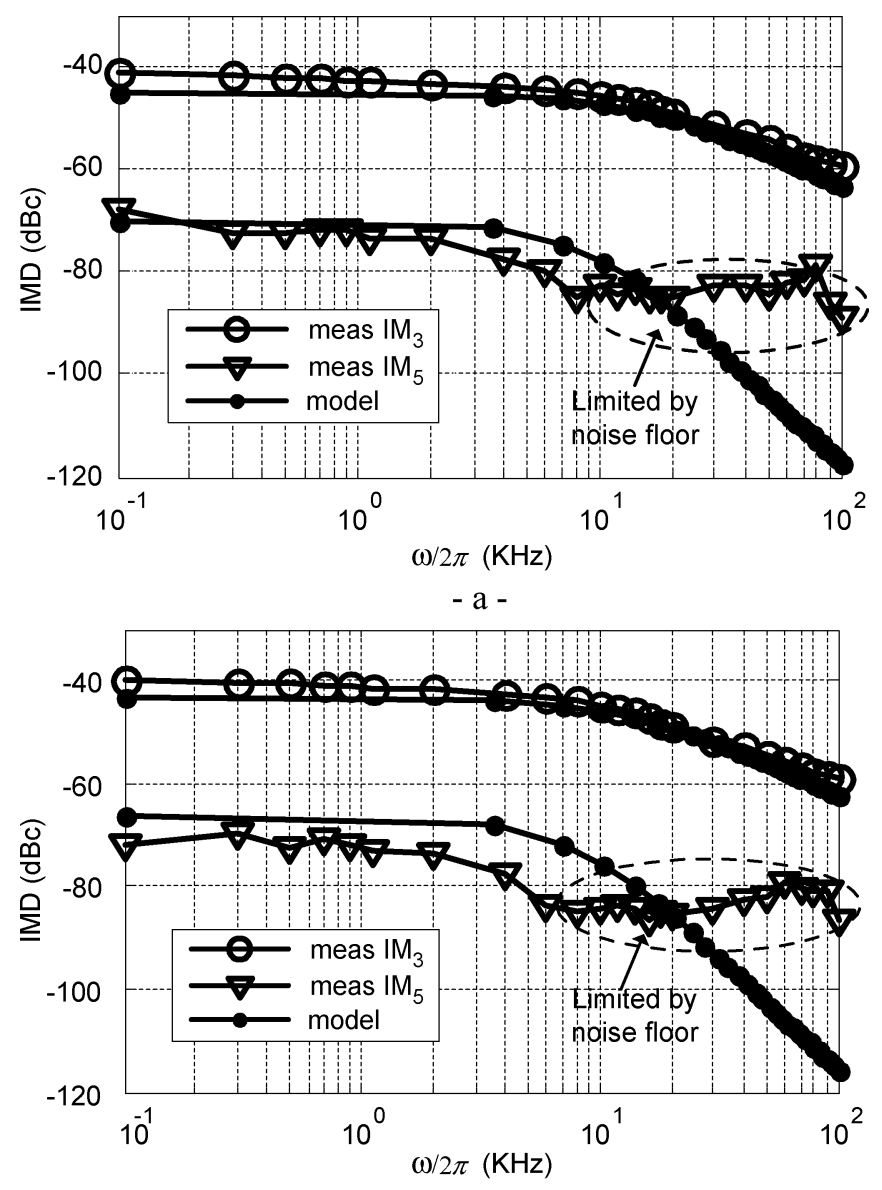

$-\mathrm{b}-$

Fig. 11. IMD as a function of $\omega$ for two biasing cases: (a) $V_{\text {bias }}=0 \mathrm{~V}$ and (b) $V_{\text {bias }}=1 \mathrm{~V}$ with $P_{\mathrm{RF}}=10 \mathrm{dBm}$.

Intermodulation increases with bias voltage, as the membrane is placed closer to the fixed electrode in a nonlinear position. This can be observed in Fig. 10, where IMD is shown as a function of the applied bias voltage $V_{\text {bias }}$ for $P_{\mathrm{RF}}=8 \mathrm{dBm}$ and $\omega / 2 \pi=100 \mathrm{~Hz}$. The model predicts a larger IMD increase due to bias voltage than the measured one, which is also attributed to the fact that the displacement sinusoid is not as much distorted as expected. The dependence of IMD on the bias voltage is also shown in Fig. 11, where two measurements of IMD as a function of $\omega$ for $P_{\mathrm{RF}}=10 \mathrm{dBm}$ and bias

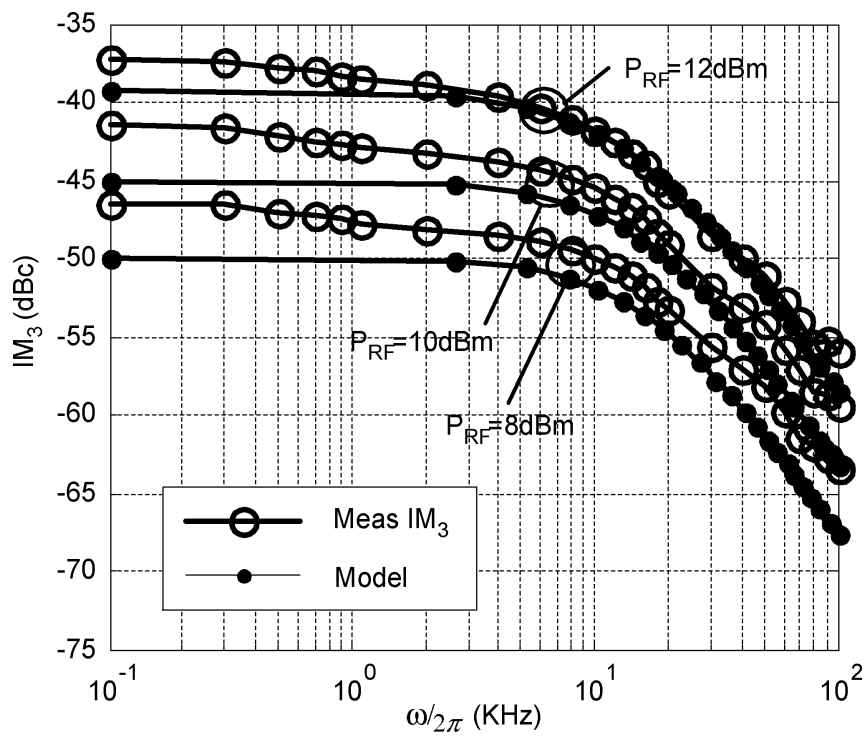

Fig. 12. Third-order IMD $\left(\mathrm{IM}_{3}\right)$. Comparison between the proposed analytical model and measurements for three RF powers $P_{\mathrm{RF}}(8,10$, and $12 \mathrm{dBm})$.

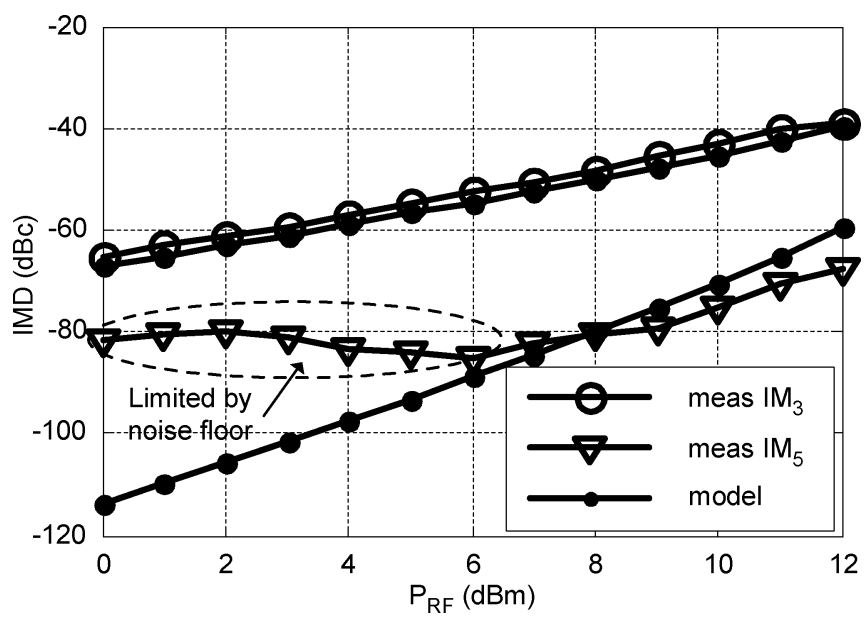

Fig. 13. Comparison between the presented model and measurements of $\mathrm{IM}_{3}$ and $\mathrm{IM}_{5}$ for the case of $\omega / 2 \pi=2 \mathrm{kHz}\left(\omega<\omega_{o}\right)$.

voltages of 0 and $1 \mathrm{~V}$ are compared to the results predicted by the analytical model.

The dependence of IMD on the applied RF power is plotted in Fig. 12, where simulations of $\mathrm{IM}_{3}$ obtained from the proposed analytical model for three RF power values are compared to measured results. An increase of $\mathrm{IM}_{3}$ with RF power, as expected, can be observed.

The evolution of IMD as a function of RF power can be more clearly observed in Fig. 13, where results of third- and fifth-order intermodulation predicted by the analytical model are compared to measurements. Fifth-order IMD is significant for RF power levels higher than approximately $+7 \mathrm{dBm}$. For lower power levels, its contribution is hidden by the noise floor. It should be noted that fifth-order IMD approaches third-order IMD for high input voltages.

The evolution of $\mathrm{IM}_{3}$ as a function of RF power for three RF tone separations $\omega$ can be observed in Fig. 14. $\mathrm{IM}_{3}$ degrades with decreasing $\omega$, as expected. 


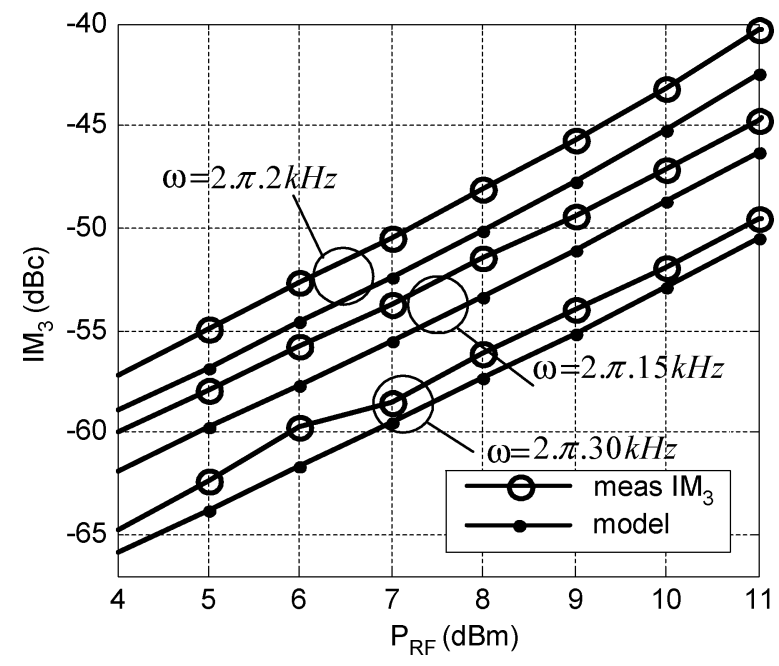

Fig. 14. $\mathrm{IM}_{3}$ as a function of the RF power $P_{\mathrm{RF}}$ for three $\mathrm{RF}$ tone separation $\omega / 2 \pi: 2,15$, and $30 \mathrm{kHz}$.

\section{DiSCUSSION ON THE RESULTS}

The need for using the total voltages $V_{1}$ and $V_{2}$ at the capacitor instead of the incident wave amplitudes $V_{01}^{+}$and $V_{02}^{+}$has been stressed in the presented formulation. This is not required in capacitive switches in the ON state since they generally have both ports terminated. This remark is important because, depending on the position of the MEMS capacitor in the circuit, the voltage at the capacitor may be different (higher or lower) from the amplitude of the incident wave, producing a higher or lower intermodulation product level. In a reflection measurement configuration, the capacitor acts as the termination of a transmission line and, thus, a standing wave is generated. The more out-of-phase incident and reflected waves are, the lower the total voltage at the capacitor is, producing a lower self-actuation and IMD, also increasing the RF current density at the device. Adding a parasitic capacity to the device-in the case of capacitors mounted in a configuration similar to the one proposed here for measurement-could be considered as a method to increase the phase shift between the incident and reflected waves and, therefore, to improve IMD behavior of MEMS capacitors.

An important result is that the measured MEMS capacitor-which has a low stiffness constant $k$ and a low initial gap $d$ (Fig. 6) - has shown low IMD figures (the worst measured values are $\mathrm{IM}_{3}=-37 \mathrm{dBc}$ and $\mathrm{IM}_{5}=-62 \mathrm{dBc}$ for the case of $P_{\mathrm{RF}}=12 \mathrm{dBm}$-near pull-in due to RF power-and $\left.\omega=100 \mathrm{~Hz}-\ll \omega_{0}\right)$, much better than its commercial semiconductor equivalents. This conclusion is remarkable since, although this study has demonstrated that IMD in MEMS capacitors is higher than in MEMS switches, this increase is not as large as could be expected from the arguments given in Section I. Moreover, it has been verified that the IMD performance in MEMS capacitors can be much improved with RF tone separations $\omega$ higher than the device mechanical bandwidth.

It must also be stressed that using a capacitor with a higher pull-in voltage does not necessarily imply that either the thirdor fifth-order IMD products disappear, but only that the same effects would shift to a higher RF power level. For this reason, the nonlinear effects and the presence of high-order IMD products studied in this paper are completely general for any two parallel-plate MEMS capacitor.

\section{CONCLUSION}

Intermodulation phenomenon in RF MEMS capacitors has been studied in depth for the general two-tone case. The roles of the various physical building parameters and external excitation conditions on IMD generation have been provided. Generation of IMD products has been analyzed and experimentally characterized by an analytical frequency-dependent model that allows a realistic IMD prediction in MEMS varactors. It has been shown that, in addition to third-order IMD products, higher order products (fifth order) emerge when the membrane is placed in the highly nonlinear regions of the tuning range (highly nonlinear dependence of the displacement on the applied voltage). These IMD products are also slightly increased due to the nonlinear dependence of the reflection-coefficient phase on the displacement. The nonlinear dependence of the displacement versus voltage contributes to the total mean position of the membrane as well. It has been demonstrated that MEMS capacitors are expected to produce more significant IMD levels than capacitive switches in spite of being considerably smaller than those generated by their commercial semiconductor equivalents. A measurement setup, based on the measurement of the reflected signal, has been proposed and used to validate the theoretical analysis, as well as the analytical model.

\section{REFERENCES}

[1] J. P. Busquère, K. Grenier, G. Bouche, D. Dubuc, P. Pons, R. Plana, and P. Ancey, "Development of a reconfigurable in frequency LNA through RF MEMS," in Proc. 5th MEMS for Millimeterw. Commun. Workshop, Jul. 2004, pp. B7-B10.

[2] G. M. Rebeiz, T. Guan-Leng, and J. S. Hayden, "RF MEMS phase shifters: Design and applications," IEEE Micro, no. 2, pp. 72-81, Jun. 2002.

[3] S. Barker and G. M. Rebeiz, "Distributed MEMS true-time delay phase shifters and wide-band switches," IEEE Trans. Microw. Theory Tech., pt. 2, vol. 46, no. 11, pp. 1881-1890, Nov. 1998.

[4] M. Behera, V. Kratyuk, S. K. De, N. R. Aluru, H. Yutao, and K. Mayaram, "Accurate simulation of RF MEMS VCO performance including phase noise," J. Microelectromech. Syst., vol. 14, no. 2, pp. 313-325, Apr. 2005.

[5] G. M. Kraus, C. L. Goldsmith, C. D. Nordquist, C. W. Dyck, P. S Finnegan, F. Austin, A. Muyshondt, and C. T. Sullivan, "A widely tunable RF MEMS end-coupled filter," in IEEE MTT-S Int. Microw. Symp. Dig., vol. 2, Jun. 2004, pp. 429-432.

[6] J. Schoebel, T. Buck, M. Reimann, M. Ulm, M. Schneider, A. Jourdain, G. J. Carchon, and H. A. C. Tilmans, "Design considerations and technology assessment of phased-array antenna systems with RF MEMS for automotive radar applications," IEEE Trans. Microw. Theory Tech., pt. 2, vol. 53, no. 6, pp. 1968-1975, Jun. 2005.

[7] L. Dussopt, "MEMS technologies for antenna applications," in Proc. 5th MEMS for Millimeterw. Commun. Workshop, Jul. 2004, pp. D6-D9.

[8] Y. Lu, L. P. B. Katehi, and D. Peroulis, "A novel MEMS impedance tuner simultaneously optimized for maximum impedance range and power handling," in IEEE MTT-S Int. Microw. Symp. Dig., Jun. 2005, pp. 927-930.

[9] G. M. Rebeiz, RF MEMS, Theory, Design, and Technology. New York: Wiley, 2003

[10] H. Nieminen, V. Ermolov, K. Nybergh, S. Silanto, and T. Ryhänen, "Microelectromechanical capacitors for RF applications," J. Micromech. Microeng., vol. 12, no. 2, pp. 177-186, 2002. 
[11] T. Vähä-Heikkilä, J. Varis, J. Tuovinen, and G. M. Rebeiz, “A reconfigurable 6-20 GHz RF MEMS impedance tuner," in IEEE MTT-S Int Microw. Symp. Dig., vol. 2, Jun. 2004, pp. 729-732.

[12] L. Dussopt and G. M. Rebeiz, "Intermodulation distortion and power handling in RF MEMS switches, varactors, and tunable filters," IEEE Trans. Microw. Theory Tech., vol. 51, no. 4, pp. 927-930, Apr. 2003.

[13] M. Innocent, P. Wambacq, S. Donnay, H. A. C. Tilmans, W. Sansen, and H. De Man, "An analytic Volterra-series-based model for a MEMS variable capacitor," IEEE Trans. Comput.-Aided Des. Integr. Circuits Syst., vol. 22, no. 2, pp. 124-131, Feb. 2003.

[14] P. Hallbjörner and J. P. Starski, "Expressions for nonlinear effects of MEMS switch beam oscillations," Electron. Lett., vol. 37, no. 11, pp. 693-694, May 2001.

[15] R. Gaddi, J. Iannacci, and A. Gnudi, "Mixed-domain simulation of intermodulation distortion in RF-MEMS capacitive shunt switches," in 33rd Eur. Microw. Conf., vol. 2, Oct. 2003, pp. 671-674.

[16] J. Johnson, G. G. Adams, and N. E. McGruer, "Determination of intermodulation distortion in a MEMS microswitch," in IEEE MTT-S Int. Microw. Symp. Dig., Jun. 2005, pp. 2135-2138.

[17] V. Rizzoli, R. Gaddi, J. Iannacci, D. Masotti, and F. Mastri, "Multitone intermodulation and RF stability analysis of MEMS switching circuits by a globally convergent harmonic-balance technique," in Proc. Eur. Microw. Assoc., vol. 1, Mar. 2005, pp. 45-54.

[18] V. Rizzoli, D. Masotti, F. Mastri, and A. Costanzo, "Nonlinear distortion and instability phenomena in MEMS-reconfigurable microstrip antennas," in Proc. 35th Eur. Microw. Conf., Oct. 2005, pp. 565-568.

[19] D. Girbau, N. Otegi, L. Pradell, and A. Lázaro, "Generation of third and higher-order intermodulation products in MEMS capacitors, and their effects," in Proc. 35th Eur. Microw. Conf., Oct. 2005, pp. 593-596.

[20] D. Girbau, A. Lázaro, and L. Pradell, "Extended tuning range RF MEMS variable capacitors using electrostatic and electrothermal actuators," in Proc. SPIE, MEMS/MOEMS Compon., and Applicat./Micromach. Microfab., vol. 5344, Jan. 2004, pp. 59-70.

[21] D. Koester, A. Cowen, R. Mahadevan, M. Stonefield, and B. Hardy, PolyMUMPS Design Handbook, Revision 10.0: MEMSCAP, 2003. [Online]. Available: http://www.memsca.com/memsrus/svcsrules.html.

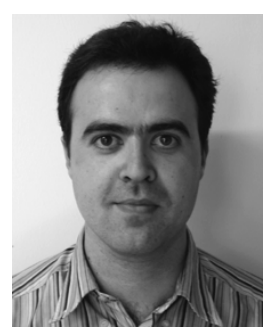

David Girbau (S'05) received the Technical Engineer in telecommunications and Engineer in Electronics degrees from the Universitat Politècnica de Catalunya, Barcelona, Spain, in 1998 and 2002 , respectively, and is currently working toward the Ph.D. degree at the Universitat Politècnica de Catalunya.

His research is currently focusing on MEMS with an emphasis on their RF and microwave applications.

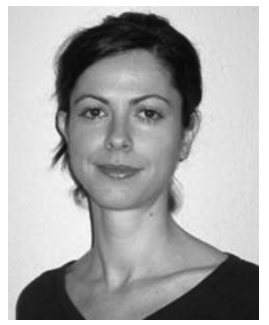

Nerea Otegi was born in Andoain, Spain, in 1977. She received the Electronic Engineering degree from the University of the Basque Country, Bilbao, Spain, in 2002, the Electronic Physics degree from the University of Cantabria, Santander, Spain, in 2003, and is currently working toward the Ph.D. degree at the University of the Basque Country.

Her areas of interest include noise characterization at microwave frequencies and nonlinear analysis of microwave circuits.

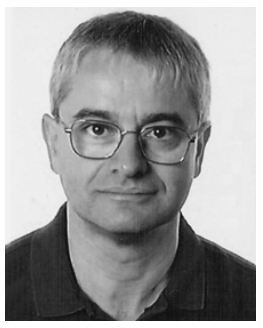

Lluís Pradell (M'87) was born in Barcelona, Catalunya, Spain, in 1956. He received the Telecommunication Engineering degree and Dr. degree in telecommunication engineering from the Universitat Politècnica de Catalunya (UPC), Barcelona, Spain, in 1981 and 1989, respectively.

From 1981 to 1985 , he was with Mier-Allende, Barcelona, Spain, as an RF and Microwave System Design Engineer. In 1985, he joined the faculty at UPC, where he became Associate Professor in 1990. Since 1985 , he has taught courses on microwave circuits and performed research on models for microwave active devices (MESFETs, high electron-mobility transistors (HEMTs), HBTs), multimodal models for guiding structures and transitions [microstrips, finlines, slotlines, coplanar waveguides (CPWs)], on-wafer measurement techniques (network-analyzer calibration, noise parameters), and development of microwave and millimeter-wave circuits (equipment for space applications, point-to-multipoint broad-band equipment, RF MEMS devices) in the frequency range of 1-75 $\mathrm{GHz}$.

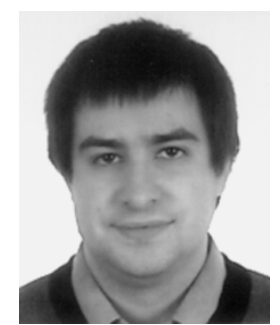

Antonio Lázaro was born in Lleida, Spain, in 1971. He received the M.S. and Ph.D. degrees in telecommunication engineering from the Universitat Politecnica de Catalunya (UPC), Barcelona, Spain, in 1994 and 1998, respectively.

He then joined the faculty at UPC, where he taught a course on microwave circuits and antennas. $\mathrm{He}$ is currently with the Electronics, Electrical, and Automatics Engineering Department, University Rovira i Virgili, Tarragona, Spain His research interests are microwave device modeling, on-wafer noise measurements, monolithic microwave integrated circuits (MMICs), low phase-noise oscillators, MEMS, and microwave systems. 